Voix et Images

voixetimages

\title{
Mario ou l'apogée du cinéma québécois
}

\section{Gilles Thérien}

Volume 10, numéro 2, hiver 1985

La barre du jour / La nouvelle barre du jour

URI : https://id.erudit.org/iderudit/200508ar

DOI : https://doi.org/10.7202/200508ar

Aller au sommaire du numéro

\section{Éditeur(s)}

Université du Québec à Montréal

\section{ISSN}

0318-9201 (imprimé)

1705-933X (numérique)

Découvrir la revue

\section{Citer cet article}

Thérien, G. (1985). Mario ou l'apogée du cinéma québécois. Voix et Images, 10(2), 215-218. https://doi.org/10.7202/200508ar d'utilisation que vous pouvez consulter en ligne.

https://apropos.erudit.org/fr/usagers/politique-dutilisation/ 


\title{
Cinéma
}

\section{Mario ou l'apogée du cinéma québécois}

\author{
par Gilles Thérien, Université du Québec à Montréal
}

Dimanche après-midi, première représentation. La salle est pratiquement pleine. Bon public: le gros sceau de pop-corn pour deux! Ils sont tous là sous la foi du battage publicitaire qui proclame Mario le film québécois de l'année, telle cette dame qui a pris la chance de venir de Boucherville sans être bien certaine qu'il y aurait une projection puisque le répondeur automatique n'en disait trop rien. Pourtant durant tout le film, beaucoup de trémoussements sur les fauteuils, une attention un peu distraite, quelques rires bien marqués, jeunes ou adultes, rarement tout le monde ensemble. Un ou deux moments d'émotion au moment où la bande sonore y incitait le plus. Qu'est-ce qui se passait dans la tête des gens? Allez donc le savoir. Je n'ai pas fait d'enquête sociologique, j'ai bien prêté l'oreille mais je n'ai rien entendu qui puisse me permettre de poser une sorte de jugement global sur la réception de ce film. Pourtant...

Mario est un film comme les souhaitent les producteurs québécois actuels: un film tiré d'un roman connu, une histoire simple et sentimentale et le moins de déploiement possible. Mario possède tous ces ingrédients. Le roman de Claude Jasmin sert de matière à scénarisation. Il raconte une histoire simple(?) entre deux frères et est tourné dans le décor enchanteur des Iles-de-la-Madeleine. Le tout donne une histoire linéraire sans grande qualité narrative et sans invention visuelle ou encore au niveau du montage. Nous reviendrons sur les moyens cinématographiques après avoir parlé de l'intrigue.

Deux frères sont liés l'un à l'autre par des liens plus forts que celui de la parenté. Mario, le plus jeune, est atteint d'un mutisme suspect et son frère Simon, un jeune adulte, s'occupe de lui. Tout le film joue sur cette relation. La rencontre d'une jeune fille, Hélène, met en danger cette relation, du moins du point de vue de Mario qui s'emploie à détruire l'amour naissant entre Simon et la jeune touriste. Il réussit, semble-t-il, mais sa condition mentale se détériore au point où il faut le mettre dans une institution. Simon ne peut accepter l'éloignement de son frère et cherche d'abord à l'empêcher en le noyant. N'ayant pu y parvenir, - on ne noie pas un adolescent de douze ans comme on noie un chat! -, il se résout à le laisser partir. Après un certain temps, il va le chercher en lui disant cette phrase énigmatique: "C'est toi qui avais raison, Mario.» Tous les deux iront ensuite se suicider d'une façon qui manque pour le moins de clarté. Toute la durée du film, Mario traîne un animal fétiche, un coyote, qui finit par jouer un rôle important dans le film, celui de l'esprit du mal ou de l'esprit «sauvage» qui habite Mario. 
Les autres personnages du film sont secondaires. Cela comprend les parents des deux enfants, la copine d'un été, les jeunes qui partagent les jeux de Mario et de Simon, le médecin... etc. Les relations avec ces personnages sont inexistantes ou à peine ébauchées. C'est le cas des parents. Les Leblanc sont, selon toute vraisemblance, des gens à l'aise. Ils possèdent l'épicerie de la place, sinon de toute l'île et le père est pêcheur de homards. Le film identifie bêtement les occupations de chacun mais ne les rassemble jamais de façon à mieux comprendre comment existe ce noyau familial, quelle est la nature de la maladie de Mario sur laquelle on ne sait finalement rien. La rencontre entre Simon et Hélène est tout simplement incroyable. C'est le coup de foudre à la seconde, le grand amour et le drame. Pas moyen de savoir si pour ces deux adolescents, il s'agit d'une première expérience ou si c'est le genre d'histoire qui se répète tous les étés. Tout le personnage de Simon est conçu de travers. Dès le début du film, Simon passe son temps à raconter à Mario une variante faible de la bataille de Roncevaux avec maintes références à Poitiers, aux Arabes et à la grande paix d'Allah où quelques esprits pervers pourraient y retrouver une autre exclamation moins romantique mais plus explicite sur les dimensions analytiques de la relation entre les deux frères. Est-ce que ce discours est une façon de pénétrer dans le cerveau de Mario? Il avoue plus loin dans le film ne pas pouvoir y parvenir. S'agit-il alors d'une sorte de délire puisé dans sa relation à son frère, délire qui le fera basculer à la fin? C'est une hypothèse de spectateur. Le film se garde bien de dire quoi que ce soit à ce sujet. En fait, c'est tout le film qui est frappé de mutisme. Les dialogues sont insignifiants et ne contribuent pas du tout à la situation narrative. Ils semblent être là pour bien indiquer qu'il ne s'agit pas d'un film muet.

Le principal reproche que l'on peut faire au film, c'est de ne pas exploiter le matériau de base qui est pourtant présent. Pourquoi Mario ne parle-t-il pas? Est-ce de naissance? Est-ce une situation qui s'est développée ultérieurement? La mère affirme à une cliente qu'il faut accepter les épreuves mais cela ne dit pas pour autant ce qui se passe, d'autant que Mario grogne avec son coyote et crie aussi lorsqu'il se sauve dans le bois ou quand il attaque Hélène. Bon, les spectateurs les plus savants pourront toujours se dire qu'il s'agit d'un cas d'autisme. Mais l'autisme ne se résume pas a ne pas pouvoir articuler de sons. Il y a aussi un comportement autistique qu'un spectateur particulièrement charitable pourrait voir se dessiner dans un certain nombre de situations mais qui ne tient pas du tout dans l'ensemble du film. L'allure générale du film repousse l'autisme du côté du fantastique, de l'imaginaire romanesque comme en témoigne cette scène de la fin où on suggère que le coyote en peluche reprend sa liberté. Le film ne parvient pas à décider de la nature de son propre récit.

Plus grave encore est l'absence d'élaboration de la relation des deux frères. De toute évidence, cette relation est marquée dans le film par l'idéologie des "grands frères», idéologie qui se répercute d'ailleurs sur la relation de Simon à toute la bande de jeunes qui gravite autour de lui de ses fantaisies arabisantes. On construit un fort au bord de la mer, on guette au 
loin les Maures, on se prépare au combat. On dirait les activités de la catholique "oeuvre des terrains de jeu» ou une version bien de chez nous des gentils organisateurs d'un Club Med. Simon est le protecteur, du moins dans la première partie du film. Lorsqu'apparaît Hélène, il se détache un peu de Mario. La relation double devient triangulaire et Mario ne peut supporter ce partage. Aussi aurons-nous droit à une séquence symbolique comme à peu près plus personne n'ose les faire: Mario découvre un nid d'oiseaux avec trois oeufs dedans. Il en prend d'abord un avec lequel il joue jusqu'à ce que l'oeuf tombe et se casse. Il ne lui reste plus qu'à empoigner les deux autres oeufs et à les écraser dans ses poings fermés. "Alea jacta est» comprend le spectateur cultivé et un peu latiniste. Ce message passé, le spectateur ne devra pas se surprendre que l'amour-passion de Simon et d'Hélène ne résiste. On voudrait toutefois aller plus profondément dans la relation des deux frères qui devient alors plus franchement incestueuse et qui se révele dans le film par le refus de Simon de laisser Mario s'endormir avec lui. Ce qui toutefois ne sera plus possible au niveau de l'amour se transformera en haine, même déguisée. Quand Simon cherche à noyer Mario pour lui éviter d'aller à l'hôpital comme il s'agissait là d'une chose impossible, alors que ce dernier vient d'être la cause de la mort d'un de ses petits copains, on peut garder l'impression que cette mort joue plutôt comme la condamnation de la relation, comme sa dénégation. Quand Simon ira chercher Mario à la fin, il reconstitue le lien mais il sait que ce lien ne pourra être maintenu que dans le suicide. C'est faire une grande charité au film de Beaudin que d'élaborer un topos où la profondeur aurait pu s'installer.

La rencontre d'Hélène et de Simon sur les plages des îles a des accents romantiques qui font penser (malheureusement) au Lagon bleu, film américain insipide mettant en vedette Brooke Shields: plages désertiques, promenades au bord de la mer, failles et crevasses invitantes et relation amoureuse dont on ne peut connaître ni l'amplitude ni la profondeur. Hélène adore celui qu'elle appelle son "arabe» et ne semble pas trop se méfier de son délire. Après une excursion où Mario fonce sur elle avec un pieu, elle disparaît du film et de la vie de Simon. Il nous faut la mère pour nous mettre sur la piste d'une éventuelle peine d'amour.

Revenons encore une fois aux Arabes. Simon organise lui-même ce jeu où il est sans cesse question de Maures. Or c'est justement de cela qu'il s'agit, de mort: les morts imaginaires du champ de bataille phantasmé, la mort accidentelle possible de Mario qui se jette à l'eau pour récupérer son coyote, la mort réelle (du moins c'est ce que le film donne à comprendre!) d'un des petits copains de jeu de Mario, la tentative de meurtre de Mario contre Hélène, celle de Simon contre Mario et finalement le double suicide. Encore une fois cette présence de la mort n'est jamais exploitée autrement que sur le plan d'une sommation d'anecdotes. Mario est un film au récit linéaire, sans profondeur qui manipule les idées de façon primaire. 
La facture du film poursuit le même dessein. L'image léchée à l'extrême transforme le film en une série de cartes postales au cadrage recherché dans un but purement esthétique. L'attention à la perspective traditionnelle avec le respect des division 1/3,2/3, l'abondance de très gros plans comme moyen unique de forcer l'émotion, les doubles foyers, les mouvements de caméra prévisibles, les zooms obligatoires, tous ces éléments contribuent à marquer le film d'une empreinte sentimentale qui détruit la profondeur du sujet. La misè en scène consiste plus à coordonner les regards et les mouvements de caméra qu'à insuffler aux comédiens quelque chose comme une âme. La manie de commencer avec ce cadre vide et de laisser l'action quitter ce cadre sent plus l'apprentissage que le procédé. Quant au montage, n'en parlons pas, il est tout aussi linéaire et peu inventif que le film. L'utilisation de la musique est grossière et nous ramène cinquante ans en arrière quand on demandait à la musique de signaler au spectateur le genre d'émotions appropriées à la scène.

L'apogée du cinéma québécois? Oui si l'on admet que le rêve premier de beaucoup de réalisateurs et de producteurs a toujours été de reconstituer ici un petit Hollywood. Mais comme il ne s'agit pas de concurrencer la Mecque du cinéma, on se contente des éternels recommencements. Nous aurons bientôt fait de former une excellente génération de réalisateurs de série B qui pourront localement maintenir une industrie dont les intérêts sont ailleurs. Il ne restera qu'à corriger les quelques défauts inévitables qui hantent les séries B comme cette ambulance qui, dans le film de Jean Beaudin, roule sur une route déserte en faisant hurler sa sirène. On a tellement peur que le spectateur ne comprenne pas... 